Wright State University

CORE Scholar

$5-1-1993$

\title{
Photoquenching and thermal Recovery of a thermally Stimulated Current Peak in Semi-insulating GaAs
}

Z-Q. Fang

David C. Look

Wright State University - Main Campus, david.look@wright.edu

Follow this and additional works at: https://corescholar.libraries.wright.edu/physics

Part of the Physics Commons

\section{Repository Citation}

Fang, Z., \& Look, D. C. (1993). Photoquenching and thermal Recovery of a thermally Stimulated Current Peak in Semi-insulating GaAs. Journal of Applied Physics, 73 (10), 4971-4974.

https://corescholar.libraries.wright.edu/physics/125

This Article is brought to you for free and open access by the Physics at CORE Scholar. It has been accepted for inclusion in Physics Faculty Publications by an authorized administrator of CORE Scholar. For more information, please contact library-corescholar@wright.edu. 


\title{
Photoquenching and thermal recovery of a thermally stimulated current peak in semi-insulating GaAs
}

\author{
Z.-Q. Fang and D. C. Look \\ Physics Department, Wright State University, Dayton, Ohio 45435
}

(Received 18 September 1992; accepted for publication 4 February 1993)

\begin{abstract}
A prominent thermally stimulated current peak $T_{5}$ appearing in semi-insulating GaAs is shown to photoquench under infrared illumination, and then thermally recover at a rate $r=2.0 \times 10^{8} \exp (-0.26 \mathrm{eV} / \mathrm{kT}) \mathrm{s}^{-1}$, exactly the same as that observed for EL2, within experimental error. Two possible explanations exist: (1) $T_{5}$ and EL2 are microscopically very similar, probably each with an $\mathrm{As}_{\mathrm{Ga}}$ core; or (2) $T_{5}$ is an electron trap that only appears to quench and recover with EL2 because EL2 controls the electron lifetime. Several other traps show similar quenching and recovery behavior.
\end{abstract}

\section{INTRODUCTION}

The midgap donor level EL2 is responsible for the semi-insulating (SI) nature of undoped GaAs. Perhaps the most prominent characteristics of EL2 are its photoquenching by strong infrared (IR) light ( $h v=1.1-1.2$ $\mathrm{eV}$ ) and then the thermal or optical reversal of this process. The experimental quantities subject to photoquenching include photoconductivity, photocapacitance, photoluminescence, $1.1 \mu \mathrm{m}$ IR absorption, and electron paramagnetic (EPR) of $\mathrm{As}_{\mathrm{Ga}}$; in each case, the photoquenching has been correlated with the transformation of EL2 from its normal state (EL2) to a metastable state $(E L 2 *)$. Because of the difficulty in detecting EL2* by direct electrical and optical means, a general consensus concerning the atomic structure of EL2 and EL2* has not been reached, although it is well accepted that EI.2 at least contains the arsenic antisite $\left(\mathrm{As}_{\mathrm{Ga}}\right){ }^{1}$ However, we and others have recently shown that thermally stimulated current spectroscopy (TSC) is able to reveal completely distinct sets of traps depending on whether EL2 is in its normal or metastable state. ${ }^{2-4}$ In particular, there is a close relationship between the EL2 $\rightleftharpoons \mathrm{EL} 2 *$ transitions, as revealed by photocurrent (PC), and the changes in the TSC spectral features during the quenching and recovery of EL2. In this article, we present convincing proof of the relationship by showing that $T_{5}$, a prominent peak near $140 \mathrm{~K}$, not only quenches in a manner nearly identical to that of the PC, but also thermally recovers at exactly the same rate as found for the $\mathrm{PC}$, within experimental error. Thus $T_{5}$, which is associated with As-rich conditions (as is EL2), either is structurally similar to EL2 (i.e., contains $\left.A s_{G a}\right)$ or has filling and emission characteristics completely controlled by the state (normal or metastable) of the EL2 defects in the sample. An auxiliary experiment shows that the latter possibility is not as likely.

\section{EXPERIMENT AND RESULTS}

Three undoped SI GaAs samples (113, 059, and 189) used in this study were cut from the centers of their respective wafers, which in turn were taken from ingotannealed crystals grown by a high-pressure liquid encapsulated Czochralski (LEC) technique using different melt stoichiometries. The EL2 concentrations, as measured by $1.1 \mu \mathrm{m}$ absorption were $1.1 \times 10^{16}, 7.4 \times 10^{15}$, and 4.0 $\times 10^{14} \mathrm{~cm}^{-3}$, corresponding to crystal stoichiometries ranging from As rich to $\mathrm{Ga}$ rich. The details of TSC spectroscopy using IR ( $h v \leqslant 1.12 \mathrm{eV}$ ) and $1.46 \mathrm{eV}$ light can be found in our previous articles. ${ }^{2,5}$ Here, we only describe the procedures for determining the thermal recovery rate of EL2. First, the sample was quickly cooled from $310 \mathrm{~K}$ to $82 \mathrm{~K}$ in the dark. At $82 \mathrm{~K}$ a strong IR light, provided by a tungsten lamp ( $25 \mathrm{~W}$ ) filtered through a Si wafer (allowing photon energies less than $1.12 \mathrm{eV}$ ) was used to illuminate the sample for $1 \mathrm{~min}$ to quench EL2. Note that because the EL2 quenching is dependent on the total dose of absorbed IR photons, the time to fully quench is determined by the intensity of the IR light, as shown in Fig. 1 of Ref. 2. Then, the sample was quickly warmed (within 1 $\min$ ) from $82 \mathrm{~K}$ up to a selected recovery temperature, $T_{r}$ $\left(110 \mathrm{~K}<T_{r}<130 \mathrm{~K}\right)$, held at that temperature for a particular waiting time, $t_{w}$ (from a few seconds to a few hours, depending on the $T_{r}$ ) and quickly cooled back to $82 \mathrm{~K}$ again. Finally, $1 \mathrm{~s}$ excitation of weak IR light (from the same tungsten lamp, but with $8 \mathrm{~W}$ output) was used to fill the traps followed by a regular thermal scan with a heating rate of $0.3 \mathrm{~K} / \mathrm{s}$ for the TSC spectrum. Our measurement procedures are very similar to the procedures used by other authors. ${ }^{6,7}$ The only difference is that we measured the charge carriers released from a particular trap, $T_{5}$, while they measured the photocapacitance and $1.1 \mu \mathrm{m}$ absorption coefficient, both of which are determined by the EL2 concentration.

Figure 1 shows the IR quenching of photocurrent $\left(I_{\mathrm{ph}}\right)$ in three samples using a strong IR light. The quenching behavior is the same as that described in Ref. 3, i.e., all behavior, including the initial $I_{\mathrm{ph}}$, the final $I_{\mathrm{ph}}$, the magnitude of $I_{\mathrm{ph}}$ quenching, and the transition time $\left(t_{T}\right)$ are stoichiometry dependent or EL2 concentration dependent. After IR quenching of EL2, i.e., $t>i_{T}$, the samples became $p$ type, as demonstrated by IR photo-Hall effect measurements at $90 \mathrm{~K}^{2,3}$ The TSC spectra before and after IR quenching of EL2 are shown in Figs. 2 and 3 for the three samples, respectively. From the figures, we find that (1) before IR quenching of EL2, seven TSC peaks $\left(T_{2}, T_{3}\right.$, $T_{4}, T_{5}, T_{5}^{*}, T_{6}$, and $\left.T_{6}^{*}\right)$, collectively designated feature I, 


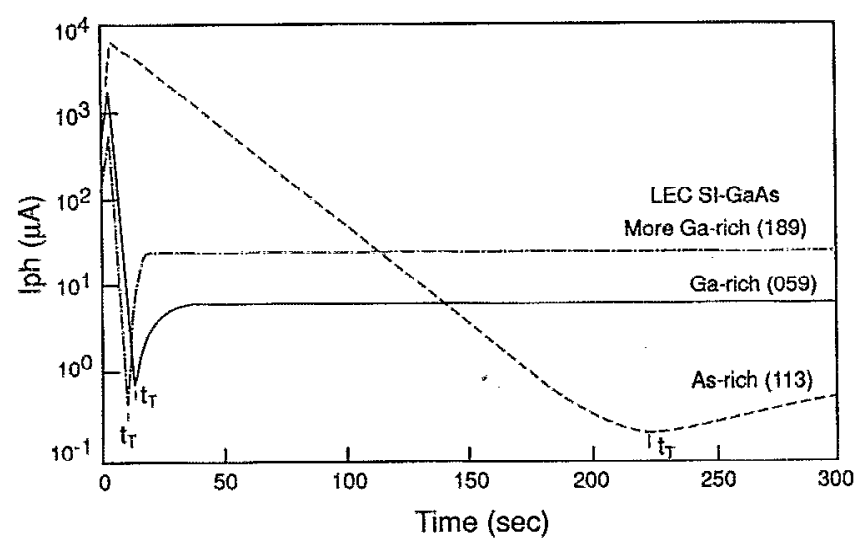

FIG. 1. Photocurrent in the presence of strong IR light excitation at 82 $\mathrm{K}$ for three LEC SI GaAs samples.

appear in the spectra; (2) the ratios of the peak heights for $T_{2}$ over $T_{3}$, and $T_{5}$ over $T_{6}$, are closely related to the crystal stoichiometry; (3) after IR quenching of EL2, the TSC spectral structure for the Ga-rich sample (059) is changed to feature II, in which five of the original peaks $\left(T_{2}, T_{3}, T_{4}, T_{5}\right.$, and $\left.T_{5}^{*}\right)$ have disappeared, and three new peaks $\left(T_{0}, T_{1}\right.$, and $\left.T_{2}^{*}\right)$ and one broad peak $\left(T_{B}\right.$, near $T_{6}$ ) have appeared; (4) in contrast to the Ga-rich sample after IR quenching of EL2, $T_{2}$ and $T_{3}$ in As-rich and more Ga-rich samples (113 and 189 ) are only partially transformed into a new peak, $T_{2}^{*}$, with $T_{2}$ and $T_{3}$ surviving, respectively; and (5) at $T>280 \mathrm{~K}$, the TSC spectra both before and after IR quenching become identical and are controlled by the dark current due to the thermal ionization of EL2.

The measurements for the thermal recovcry rate of $T_{5}$ were performed on the Ga-rich sample (059), since the sample shows a complete $T_{5}$ peak without any oscillation both during the IR quenching and the thermal recovery processes. Such oscillations or "spikes" have been observed in the As-rich sample (113) and some SI GaAs samples

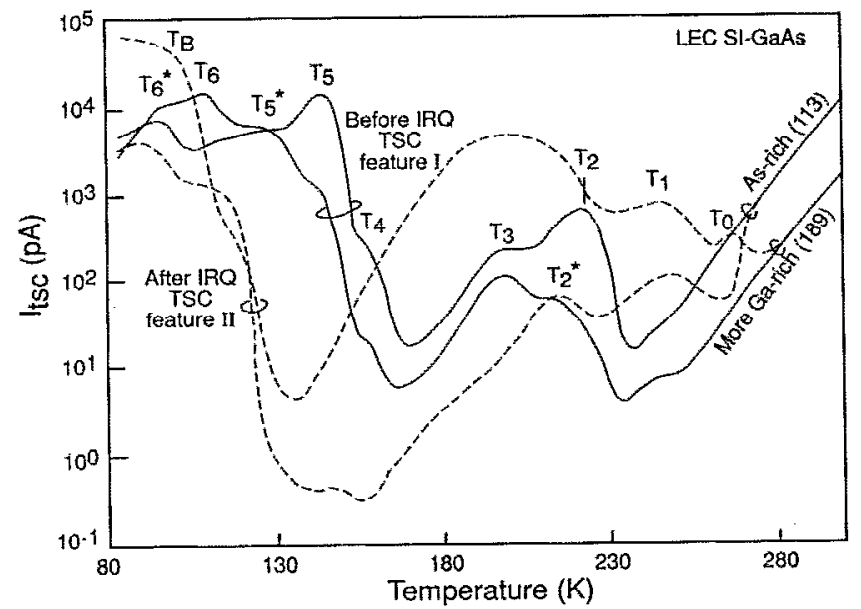

FIG. 2. TSC spectra before and after IR quenching of EL2 for As-rich (113) and more Ga-rich (189) samples.

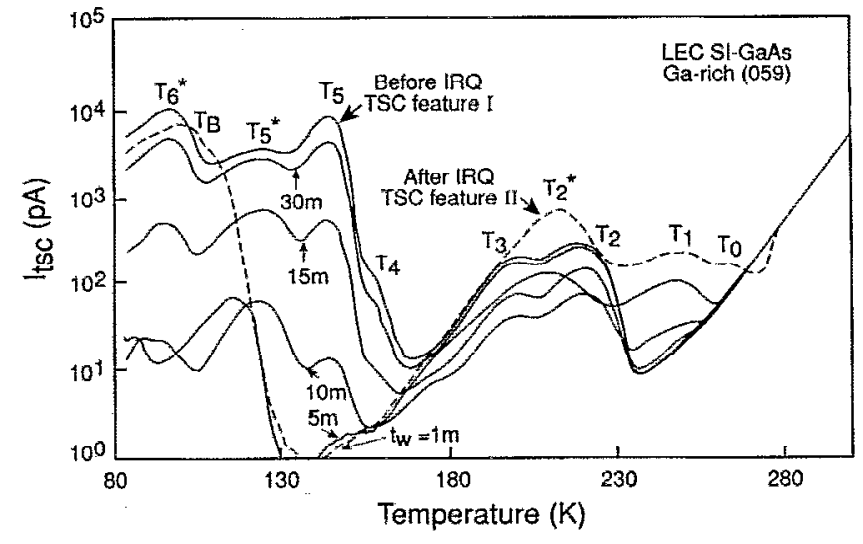

FIG. 3. TSC spectra, using $1 \mathrm{~s}$ IR (weak) excitation at $82 \mathrm{~K}$, as a function of waiting time, $t_{w}$, at $T_{r}=115 \mathrm{~K}$ with a strong IR quenching at $82 \mathrm{~K}$ for each run on a Ga-rich sample (059).

grown by the vertical gradient freeze (VGF) technique. 3.5 Typical TSC spectra as a function of $t_{w}$ with a selected $T_{r}$ of $115 \mathrm{~K}$ are shown in Fig. 3. It can be seen that with increasing $t_{w}$ from 1 to $60 \mathrm{~min}$, the TSC spectrum is gradually changed from feature II back to feature I, corresponding to the transition of EL2* $\rightarrow$ EL2. This phenomenon was also observed by changing $T_{r}$ alone. ${ }^{2,3}$

Plots of the peak heights of $T_{5}$ as functions of waiting time $t_{w}$ for various recovery temperatures $T_{r}$ are shown in Fig. 4. To model this process, let us assume that the $T_{5}$ peak disappears during IR quenching because the normal state $T_{5}^{0}$ goes to a metastable state $T_{5}^{*}$, of concentration $N_{5}^{*}$, and then $T_{5}^{0}$ reappears during heating because of the thermally induced reaction $T_{5}^{*} \rightarrow T_{5}^{0}$. If the latter reaction involves a barrier of energy $E_{b}$, as is usually assumed in such cases, then the reaction kinetics are relatively simple:

$$
\frac{d N_{5}^{*}}{d t_{w}}=-v_{0} e^{-E_{b} / k r_{r} N_{5}^{*}}
$$

or

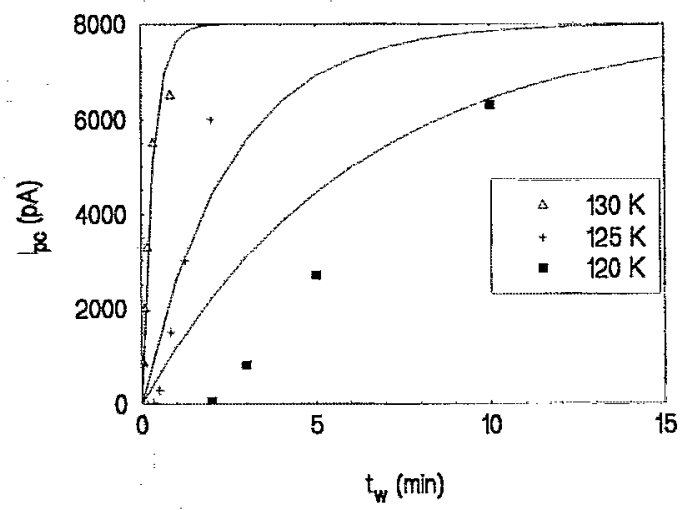

FIG, 4. Peak height of $T_{S}$ vs waiting time at different recovery temperatures, $T, s$. Solid lines are fitted by using the parameters given in Table 1 . 
TABLE I. Parameters determined by least-squares fitting of Eq. (3) to the data of Fig. 4.

\begin{tabular}{lll}
\hline \hline$T_{r}$ & $\nu_{i j}\left(\mathrm{~s}^{-1}\right)$ & $E_{b}(\mathrm{eV})$ \\
\hline 110 & $1.8 \times 10^{8}$ & 0.281 \\
115 & 2.0 & 0.271 \\
120 & 2.0 & 0.264 \\
125 & 2.0 & 0.260 \\
130 & 1.9 & 0.247 \\
\hline
\end{tabular}

$$
N_{5}^{*}=N_{5}^{*}\left(t_{w}=0\right) \exp \left[-v_{0} e^{-E_{b^{\prime}} / k r_{r_{w}}}\right] .
$$

Since $N_{5}=N_{5}^{0}+N_{5}^{*}$ we have

$$
N_{5}^{0}=N_{5}\left[1-\frac{N_{5}^{*}\left(t_{w}=0\right)}{N_{5}} \exp \left(-v_{0} e^{\left.\left.-E_{b^{\prime}} k T_{r} t_{w}\right)\right] .}\right.\right.
$$

Using a general least-squares technique, we have fitted Eq. (3) to the data of Fig. 4 under the assumption $N_{5}^{*}\left(t_{w}\right.$ $\equiv 0) / N_{5}=1$, i.e., all of the $T_{5}$ centers are quenched by the IR irradiation. The best-fit theoretical curves are shown by solid lines with the fitting parameters given in Table I. The first thing to note is that $v_{0}$ is remarkably constant, given the several orders of magnitude variation in both the $T_{5}$ peak height and the waiting time $t_{u \text {. }}$ Even $E_{b}$ varies less than $7 \%$ around the value $0.26 \mathrm{eV}$, although the variation is systematic (about $-2 \times 10^{-3} \mathrm{eV} / \mathrm{K}$ ) and may indeed be real. The second thing to note is that Mohapatra and $\mathrm{Ku}$ mar $^{8}$ have studied photoquenching of the photoconductivity in SI GaAs and found that $v_{0}=2.5 \times 10^{8} \mathrm{~s}^{-1}$ and $E_{b}$ $\equiv 0.26 \mathrm{eV}$, exactly the same as our values, within experimental error. Since the photoconductivity is known to be controlled by EL2, we can conclude that EL2 and $T_{5}$ are governed by nearly the same recovery kinetics.

The most obvious conclusion from these results might be that $T_{5} \equiv$ EL2. However, such an assertion does not necessarily follow, as evidenced by the fact that we have found the same $v_{0}$ and $E_{b}$ for a center in molecular-beam epitaxial GaAs grown at $400^{\circ} \mathrm{C}$, which we can conclusively show is not EL2. ${ }^{9}$ The most likely explanation is that each of these centers contains $\mathrm{As}_{\mathrm{Gia}}$, and that the recovery kinetics are determined mainly by the $\mathrm{As}_{\mathrm{Ga}}$ itself and not by its immediate surroundings. Another reason why $T_{5}$ is probably not identical to EL2 is that the $T_{5}$ electronic transition energy is $0.27 \mathrm{eV}$, as reported earlier ${ }^{10}$ and confirmed below, whereas the main transition in EL2 is at about $0.75 \mathrm{eV}$.

The activation energies $\left(E_{T}\right.$ 's $)$ for the main traps observed before and after IR quenching of EL2 were carefully determined on sample 059 using a thermal cleaning technique. ${ }^{\prime}$ In this technique, the $E_{T}$ for a given trap is determined from an initial-rise slope or low-temperature exponential edge, which is measured after removing all emissions from traps shallower than the test one by raising the temperature to a selected waiting temperature $T_{w}\left(T_{w}\right.$ $<T_{m}$, the peak temperature for a given trap), waiting for a long time ( $\geqslant 10 \mathrm{~min})$, and starting the thermal scan again from $82 \mathrm{~K}$. The TSC spectrum and the initial-rise slopes are shown in Figs. 5(a) and 5(b), which were measured
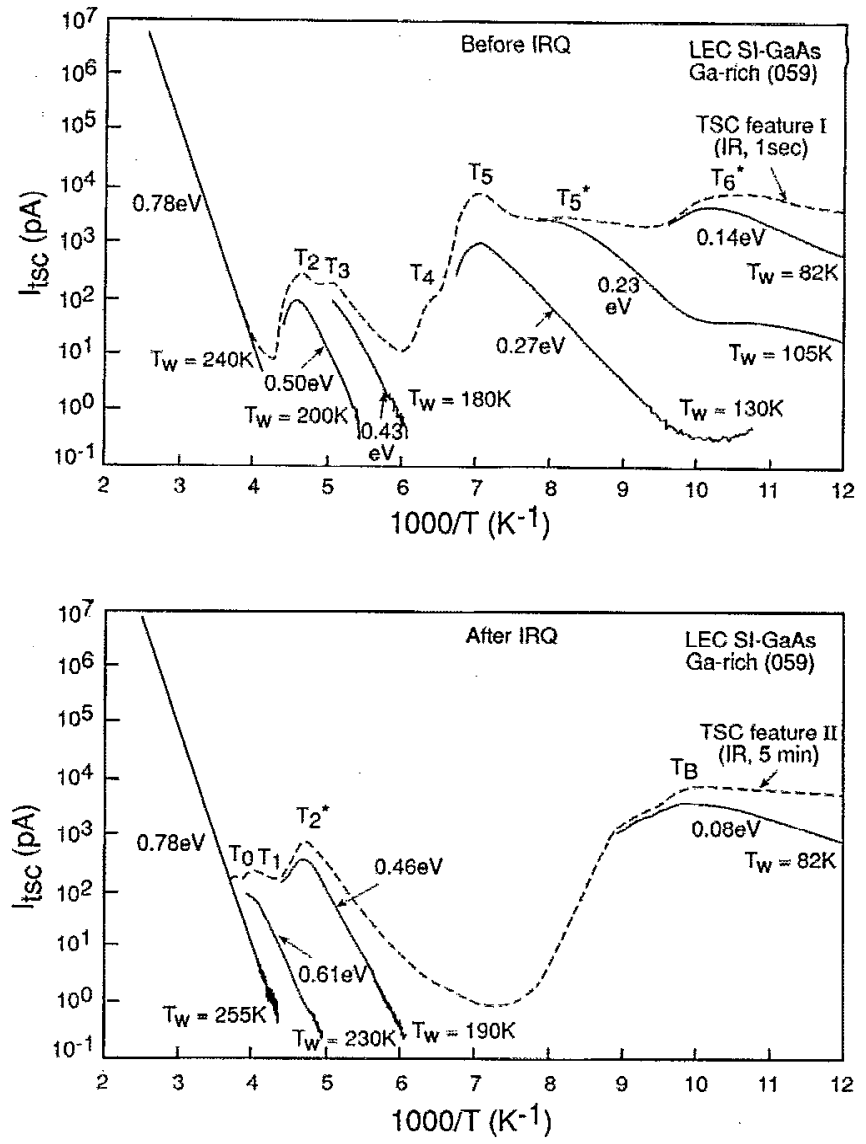

FIG. 5. Activation energies $\left(E_{T}\right.$ 's) for the main traps, determined by the thermal cleaning technique, both before (a) and after (b) IR quenching of El2.

using $1 \mathrm{~s}$ and $5 \mathrm{~min}$ weak IR light excitation at $82 \mathrm{~K}$, respectively, producing to the normal and metastable states of EL2. The $E_{T}$ 's associated with EL2 in its normal state are $0.50 \mathrm{eV}\left(T_{2}\right), 0.43 \mathrm{eV}\left(T_{3}\right), 0.27 \mathrm{eV}\left(T_{5}\right), 0.23 \mathrm{eV}$ $\left(T_{5}^{*}\right)$, and $0.14 \mathrm{eV}\left(T_{6}^{*}\right)$, while the $E_{T}$ 's associated with EL2 in its metastable state are $0.08 \mathrm{eV}$ (broad $T_{B}$ ), 0.46 $\mathrm{eV}\left(T_{2}^{\star}\right)$, and $0.61 \mathrm{eV}\left(T_{1}\right)$. The $E_{T}$ 's for $T_{4}$ and $T_{6}$ (two shallower traps) were calculated by using an approximate equation, $E_{T}=k T_{m} \ln T_{m}^{4} / \beta$ (Ref. 12) to be $0.29 \mathrm{eV}\left(T_{4}\right)$ and $0.21 \mathrm{eV}\left(T_{6}\right)$. In this equation, $k$ is Boltzmann's constant and $\beta$ is the heating rate during the thermal scan. With a capture cross section correction, ${ }^{12}$ the $E_{T}$ for $T_{0}$, a deeper trap was calculated to be $0.72 \mathrm{eV}$.

\section{DISCUSSION}

We have shown that a prominent TSC peak $T_{5}$ quenches and thermally recovers in a manner remarkably similar to that of EL2. Moreover, several other TSC peaks appear to also have the same quenching and recovery properties, although we have not precisely fitted their kinetics. The most likely explanation for these phenomena is that each of the centers represented by these TSC peaks contains a common element as a core, most likely the arsenic antisite $\mathrm{As}_{\mathrm{Ga}}$. However, we must also consider a second possibility, as outlined below. 
The observation of a TSC peak requires (1) that free electrons or holes be available to fill the trap in question, and (2) that the electrons or holes emitted during the heating cycle have a lifetime long enough to give a measurable current. Obviously both these conditions are fulfilled for $T_{5}$ if EL2 is not quenched, because $T_{5}$ is a prominent peak under such circumstances. But the question remains as to whether either of these conditions could be violated if EL2 were quenched. For example, if the IR light excitation to fill the traps mainly produced electrons via excitation from EL2 in its normal state, then no electrons would be available if EL2 were in its metastable state (i.e., quenched), and electron traps could not be filled. To eliminate this possibility, we flooded the sample in the quenched state with both $1.46 \mathrm{eV}$ monochromatic light and with white light, thus producing electrons from the valence band, and still a TSC peak for $T_{5}$ was not observed. The second possibility, a very short carrier lifetime, is harder to eliminate. For example, we could argue that if EL2 is in its quenched state, then any excited electrons recombine immediately and are not likely to be trapped, or even if they are trapped, they recombine quickly upon emission and thus produce a very small current. Support for a possible short electron lifetime comes from the fact that the samples are $p$ type after quenching, and thus the electrons have many more empty recombination sites, as well as free holes, available.

At this point, we cannot definitely say that the various traps that quench and recover with EL2 are all similar in microscopic structure, because we cannot eliminate the possibility that a short electron lifetime in the quenched state precludes trap filling or a significant current during emission. If any of the traps observed in the quenched state could be unambiguously shown to be electron traps, then the short-lifetime explanation would be in doubt. In this regard, it should be noted that $T_{6}^{*}$, which is known from electron-irradiation experiments to be an As-vacancyrelated electron-trap level, ${ }^{3,13}$ appears to exist in the quenched state (see Fig. 2). However, more detailed studies will need to be carried out to determine if this is indeed the case.

Another question concerns the traps, such as $T_{0}$ and $T_{1}$, which appear only in the quenched state. Are they associated with various complexes of the metastable $\mathrm{As}_{\mathrm{Ga}}$, or are they simply hole traps that cannot be observed in unquenched samples because the hole lifetime is too short? All of these questions must await further study, especially attempts to positively identify the electron- or hole-trap nature of the various TSC peaks.

\section{CONCLUSIONS}

For the first time, a thermally stimulated current peak, $T_{5}$, near $140 \mathrm{~K}$, has been shown to quench under IR illumination and then thermally recover with exactly the same kinetics as those of EL2, within experimental error. There are two possible explanations for this observation: (1) $T_{5}$ and EL2 are microscopically very similar, probably with regard to an $\mathrm{As}_{\mathrm{Ga}}$ core in each case, or (2) the peak height of $T_{5}$ is controlled by the electron lifetime, which in turn is controlled by EL2 either directly or via the Fermi level. Further studies will be necessary to solve this problem unambiguously.

\section{ACKNOWLEDGMENTS}

We wish to thank J. W. Look for carrying out the least-squares fitting analysis of Eq. (3) to the data of Fig. 4 , and N. Blair for manuscript preparation. The work was supported by ONR Contract NOO014-90-J-11847 and by a grant from the Nippon Mining Company, Japan.

${ }^{1}$ M. O. Manasreh, D. W. Fischer, and W. C. Mitchel, Phys. Status Solidi B 154, 11 (1989).

${ }^{2}$ Z.-Q. Fang and D. C. Look, Appl. Phys. Lett. 59, 48 (1991).

${ }^{3}$ Z.-Q. Fang and D. C. Look, Mater. Sci. Forum Vol. 83-87, 991 (1992).

${ }^{4}$ U. V. Desnica, D. I. Desnica, and B. Santic, Appl. Phys. Lett. 58, 278 (1991).

${ }^{5}$ Z.-Q. Fang and D. C. Look, J. Appl. Phys. 69, 8177 (1991).

${ }^{6}$ A. Mitonneau and A. Mircea, Solid State. Commun. 30, 157 (1979).

${ }^{7}$ D. W. Fischer, Phys. Rev. B 37, 2968 (1988).

${ }^{8}$ Y. N. Mohapatra and V. Kumar, J. Appl. Phys. 64, 956 (1988).

${ }^{9}$ D. C. Look, Z.-Q. Fang, J. R. Sizelove, and C. E. Stutz, Phys. Rev. Lett. 70, 465 (1993).

${ }^{10}$ Z.-Q. Fang, L. Shan, T. E. Schlesinger, and A. G. Milnes, Mater. Sci. Eng. B 5, 397 (1990).

"A. G. Milnes, Deep Impurities in Semiconductors (Wiley, New York, 1973) p. 236.

${ }^{12}$ D. C. Look, Semiconductors and Semimetals (1983), Vol. 19, p. 75.

${ }^{13}$ Z.-Q. Fang, J. W. Hemsky, and D. C. Look, Bull. Amer. Phys. Soc. 37, 251 (1992). 